\title{
1. Development of a Human Physiologically Based Pharmacokinetic (PBPK) Model for Dermal Permeability for Lindane
}

\author{
Megan E. Sawyer ${ }^{\mathrm{a}, 1, *}$, Marina V. Evans ${ }^{\mathrm{b}}$, Charles A. Wilson ${ }^{\mathrm{c}}$, Lauren J. Beesley ${ }^{\mathrm{c}}$, Lider S. Leon ${ }^{\mathrm{c}}$, Chris \\ R. Eklund ${ }^{\mathrm{b}}$, Edward L. Croom ${ }^{\mathrm{b}}$, Rex A. Pegram ${ }^{\mathrm{b}}$ \\ ${ }^{a}$ Department of Mathematics, Campus Box 8205, North Carolina State University, \\ Raleigh, NC 27695, USA \\ ${ }^{b}$ National Health and Environmental Effects Research Laboratory, US Environmental Protection Agency, Office of Research \\ and Development, Research Triangle Park, NC 27709, USA \\ ${ }^{c}$ Research Experience for Undergraduate participant, Department of Mathematics, North Carolina State University, Raleigh, \\ NC 27695, USA
}

${ }_{11}$ Abstract

* Corresponding Author

Email address: m.sawyer@snhu.edu (Megan E. Sawyer)

${ }^{1}$ Present Address: Department of Mathematics, Southern New Hampshire University, Manchester, NH 03106, USA; Phone: $1-(603)-668-2211$

Preprint submitted to Toxicology Letters

December 3, 2015

(C) 2016. This manuscript version is made available under the Elsevier user license http:/www.elsevier.com/open-access/userlicense/1.0/ 
- HT: High-throughput

- SC: Stratum corneum

\section{Introduction}

The US Environmental Protection Agency (US EPA) has the responsibility to determine human health risk from exposure to chemical compounds that may be environmental contaminants. However, limited financial resources are available to perform traditional toxicological screens on all potentially toxic chemicals. With the development of efficient high-throughput (HT) in vitro techniques, a strategy has been created to correlate toxicity with HT measurements. This has become the US EPA's ToxCast research program, motivated by a chemical prioritization framework flexible enough to incorporate new data as they become available [1]. Since it is not feasible to obtain experimental data for all chemicals, this program also relies on predictive toxicology methodology, where chemicals are prioritized for further testing based on physicochemical characteristics and their potential for biological disruption of key pathways. The US EPA National Center for Computational Toxicology (NCCT) has already conducted essential research needed to correlate chemical characteristics and biological activity as listed in the ToxCast database, developed in phases and currently containing over 2,000 chemicals. An integrative modeling framework will become essential in this predictive toxicology paradigm - one that integrates exposure, dose, and toxicity for many chemicals. Up to this point, physiologically based pharmacokinetic (PBPK) models have been used to convert environmental exposure to an internal (target) dose for a single chemical at a time. As the application of PBPK modeling has become more accepted, a trend has emerged to include PBPK modeling as part of an overall computational strategy. Current efforts to merge PBPK modeling with exposure modeling describing populations use different routes of exposure [2]. Dermal exposure is one of the most common types of exposure, particularly for pesticides, water-borne chemicals, and consumer products.

Lindane was used as an agricultural insecticide until December 2006, when the US Environmental Protection Agency canceled registration of agricultural products containing this chemical [3]. This decision was due in part to the negative effect on the central nervous system: lindane binds to GABA receptors, 
leading to increased excitability of these neurons $[4,5]$. This binding causes both acute and chronic effects such as convulsions and seizures even at low doses [6]. Recently, lindane has been upgraded to a human carcinogen by IARC 7]. Prior to this, the US Food and Drug Administration approved the use of a $1 \%$ lindane solution for the treatment of head lice and scabies [8], albeit with a warning about potential toxicity and a recommendation for use only as a second tier treatment. Due to its use in humans as a therapeutic agent, particularly children, extensive studies have been conducted to determine the time course of lindane disposition. To produce data relevant to pharmaceutical skin applications, human kinetic studies have used dermal exposure as the entry route for lindane [9]. Availability of a human dermal database and its potential toxicological effect on different life stages and populations make lindane an excellent choice for a modeling study.

The skin is a complex organ consisting of stacked layers composed of a combination of lipophilic and protein-rich tissues. Compartmental models simulating different layers have been used to describe a chemical's penetration [10, 11]. Dermal penetration is a function of both the chemical's properties such as lipophilicity, and the composition of the application vehicle. Despite the biological complexity, penetration can be modeled using the fundamental diffusion process (Fick's law). This physical law indicates that movement of the chemical across the skin is proportional to the gradient in chemical concentration, where the proportionality constant is the diffusion constant. The goals of the present work were: 1) to obtain estimates for the dermal diffusion constant based on physicochemical and biochemical properties, 2) to use a PBPK model for lindane in combination with an in vivo dataset to optimize for the dermal diffusion constant in humans, and 3) to find a method to calculate dermal absorption which could be generalized to other ToxCast compounds.

\section{Methods}

\subsection{Experimental human data}

In vivo time-course data were obtained from published literature [9]. Dermal absorption of lindane in a white spirit vehicle (indicated as Formulation A) was measured in blood plasma at fixed time points after 
94 Eq. 2.2 as described by [13]:

95

96 and

\subsection{Dermal constants} Potts-Guy [12] equation:

exposure on the forearms of several volunteers. Six hours post-exposure, the skin was wiped of all remaining lindane. Venous blood samples were taken from the unexposed arm until 80 hours post-exposure and plasma samples were analyzed for lindane concentrations. The experiment utilized a finite dosing scheme; no additional chemical was applied to the surface of the skin as absorption occurred.

This study utilized a homogenous compartmental model assuming both a well-mixed vehicle compartment and a well-mixed skin compartment. Diffusion is the main mechanism governing penetration of the chemical into the skin through the stratum corneum (SC) into the main dermal compartment. This can be mathematically described using Fick's one-dimensional diffusion equation:

$$
\frac{\partial C}{\partial t}=D\left(\frac{\partial^{2} C}{\partial l^{2}}\right) .
$$

Here, $C$ is the chemical concentration, $D$ is the diffusion constant, and $l$ represents the diffusional distance of the chemical (here, assumed to be skin depth). In addition, the solubility between the vehicle and the SC is assumed to be constant, and is given as the dermal partition coefficient $P_{d e r}$. This constant is different for each chemical and is calculated as described below.

The default method used by the US EPA for calculating the permeability coefficient $K_{p}$ is given by the

$$
\log K_{p}=-2.8-6.0 \cdot 10^{-3} \cdot(M W)+0.74 \cdot \log P_{\text {ow }} .
$$

This permeability equation can also be modified to predict the dermal partition coefficient $P_{\text {der }}$ by separating

$$
\log P_{d e r}=0.74 \cdot \log P_{o w}
$$

97

$$
\log \left(\frac{D}{l}\right)=-2.8-6.0 \cdot 10^{-3} \cdot(M W)
$$


However, binding to plasma proteins, particularly albumin, is not incorporated into Eq. 2.2. As an adaptation, this study utilized equations in [14] to calculate the following: the vehicle:water $\left(P_{v: w}\right)$, SC:water $\left(P_{s c: w}\right)$, and dermis:water $\left(P_{d e r: w}\right)$ partition coefficients; the vehicle:dermal partition coefficient $P_{d e r}$; the diffusion constant $D$; and the permeability coefficient $K_{p}$. The following equations (Eq. 2.5 Eq. 2.7) are used to calculate the partition coefficient between the vehicle and the dermis, $P_{\text {der }}$ (Eq. 2.8):

$$
\begin{aligned}
P_{v: w} & =4.62 \cdot P_{o: w}^{0.55}, \\
P_{s c: w} & =\frac{\rho_{\text {lipid }}}{\rho_{\text {water }}} \cdot P_{o w}^{0.69}, \\
P_{\text {der: }: w} & =0.7\left(0.68+\frac{0.32}{f_{u}}+0.025 \cdot P_{s c: w}\right), \\
P_{\text {der }} & =P_{\text {der }: w} / P_{v: w} .
\end{aligned}
$$

Here, $P_{o w}$ is the chemical octanol:water partition coefficient, $\rho_{\text {lipid }}$ is lipid density, $\rho_{w a t e r}$ is water density, and $f_{u}$ represents the fraction of lindane not bound to albumin [14]. Taking into consideration binding to albumin, the generalized diffusion constant is as follows:

$$
D\left(\mathrm{~cm}^{2} / \mathrm{hr}\right)=\left[\frac{10^{(-8.15-0.655 \cdot \log (M W))}}{0.68+\frac{0.32}{f_{u}}+0.025 \cdot P_{s c: w}}\right] \cdot 3.6 \times 10^{7}
$$

During the steady-state portion of dermal exposure, the relationship between different constants becomes linear with respect to each other. This in turn, makes calculation of these constants from experimental data easier. These relationships among constants have been summarized by [15]:

$$
K_{p}(\mathrm{~cm} / \mathrm{hr})=\frac{P_{d e r} D}{l}
$$

where $K_{p}$ is the permeability coefficient and $l$ is the diffusional path length. 


\subsection{In Vivo PBPK Model}

A published flow-limited in vivo human PBPK model [16] was modified through the inclusion of a skin patch and a media compartment. This skin patch was utilized as the location for the chemical exposure; the remaining portion of the skin was incorporated into the slowly perfused compartment. The PBPK model was coded and solved using MATLAB ${ }^{\circledR}$, version 8.2.0.701 (R2013b). A diagram, equations, and parameter values for the model can be found in Supplemental Material. The partition coefficient calculation obtained using Eq. 2.8 was fixed for all simulations. An initial guess for the diffusion constant $D$ was estimated using Eq. 2.9. The in vivo PBPK model and in vivo plasma time course data were combined to estimate a final value for $D$. The optimization process was performed using the function fminsearch, where cost of fit was defined to be a linear least-squares problem.

\section{Results}

Partition coefficients, the diffusion constant, and the permeability coefficient were calculated with equations that incorporated albumin binding (Eq. 2.5 Eq. 2.10). This study fixed the vehicle:dermal partition coefficient constant and estimated $K_{p}$ by varying the diffusion constant $D$. A summary for the initial and optimized values for these parameters can be found in Table 1. Within this table, cost is defined to be the sum of the residuals, as calculated using linear least squares.

Table 1: Calculation for in vivo dermal constants using Eq. 2.8 Eq. 2.10

\begin{tabular}{lll}
\hline Parameter & Initial & Final \\
\hline$P_{\text {der }}$ & 1.2 & 1.2 \\
$D\left(\mathrm{~cm}^{2} / \mathrm{hr}\right)$ & $3.77 \times 10^{-4}$ & $3.32 \times 10^{-5}$ \\
$K_{p}(\mathrm{~cm} / \mathrm{hr})$ & $1.52 \times 10^{-3}$ & $1.33 \times 10^{-4}$ \\
Cost & 55.6 & $5.37 \times 10^{-2}$ \\
\hline
\end{tabular}

The resulting time course model predictions using post-optimized values from Table 1 is shown in Fig. 1 Here, the measured mean plasma concentrations experimental data are denoted with an asterisk and the error bars present the standard error of the mean for $n=4$ subjects $[9]$. The sudden change in the slope at 


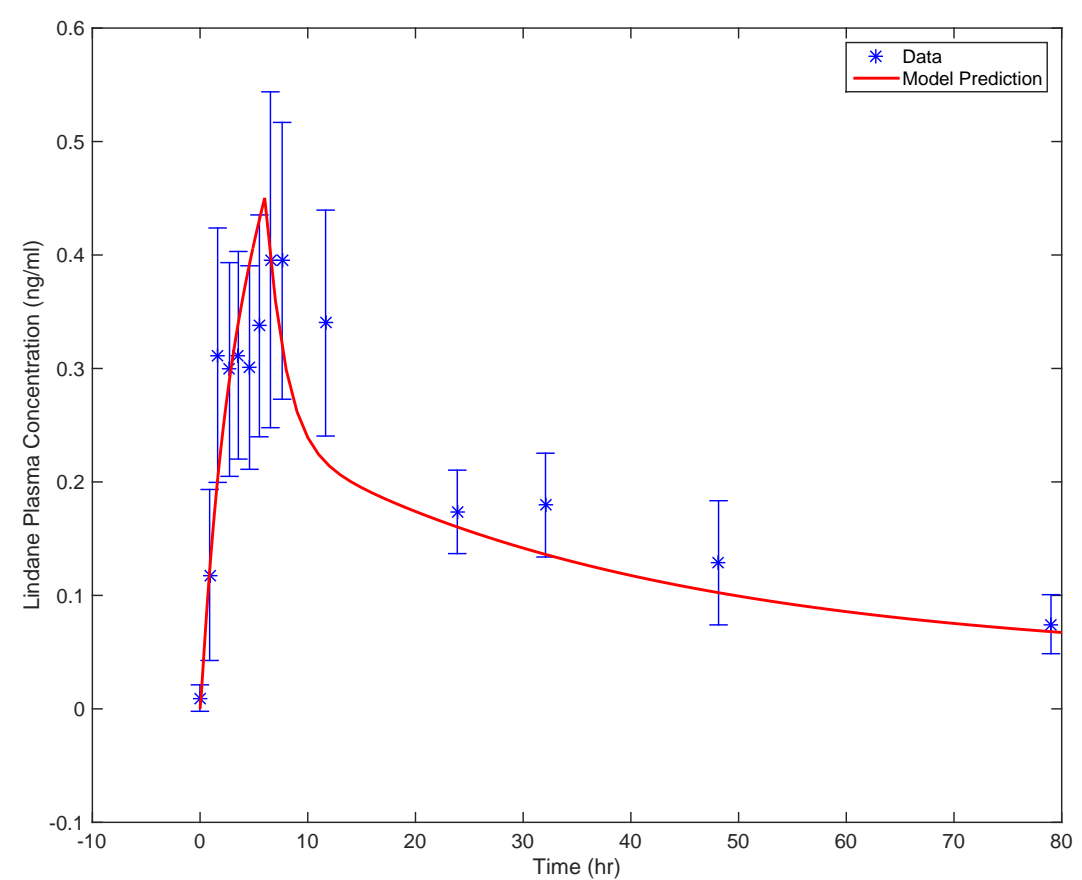

Figure 1: In vivo plasma concentration using optimized parameters, as given in Table 1 Asterisks indicate mean value of data with error bars presenting s.e.m. for $n=4$ subjects

$t=6$ hours corresponds to removal of lindane from the surface of the skin. Varying the optimized value of $D$ by $\pm 10 \%$ changes the model predictions in an expected way (Fig. 2 .

\section{Discussion}

PBPK models can be used to describe a variety of exposure routes (e.g., inhalation, oral, intravenous, or dermal). We chose a pesticide/pharmaceutical having exposure data in several routes for humans. The extensive dataset allows for comparison across different exposure routes using the same PBPK model, increasing our confidence in parameter values when fits across routes can be obtained using the same PBPK parameters as described in [16]. As lindane is still used as a topically-applied pharmaceutical compound for lice or scabies, lindane kinetics have been extensively studied in humans. This kinetic dataset allows for the corroboration of an aggregate PBPK model directly in humans, making it an ideal choice for testing a generic approach that will include compounds having less kinetic information. 


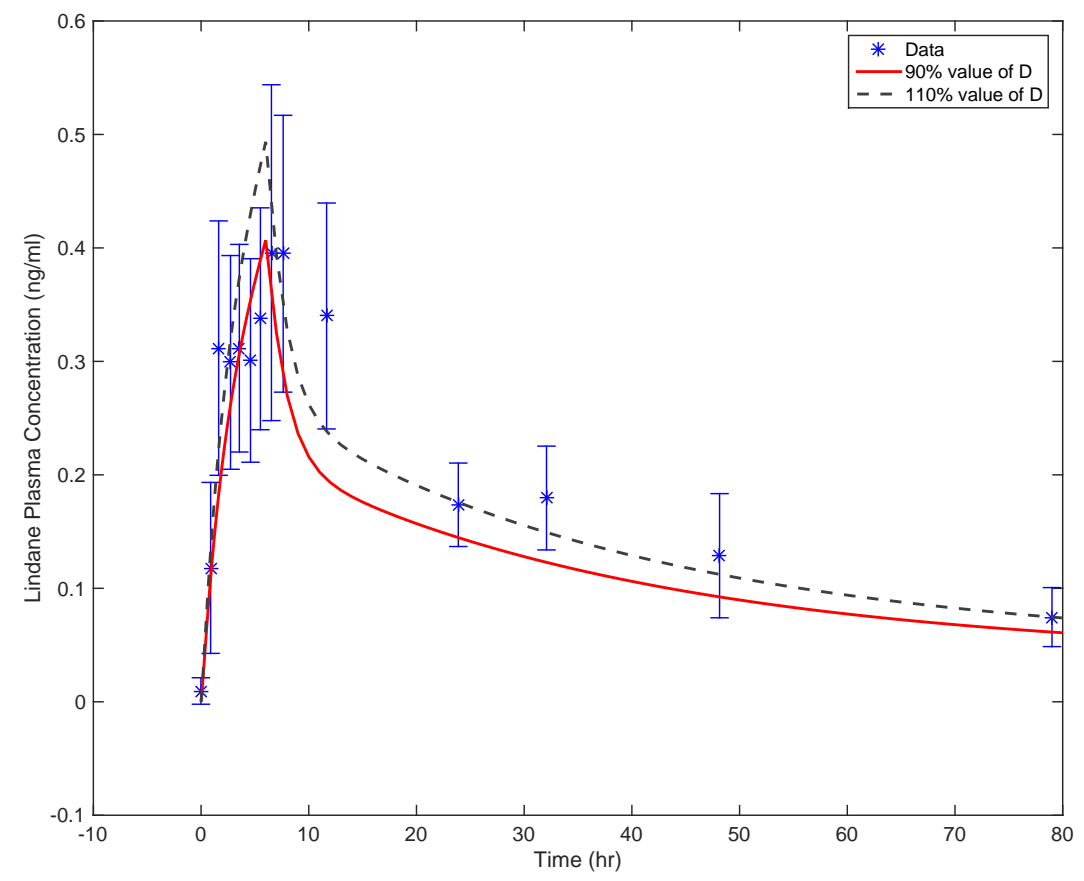

Figure 2: Model predictions with $D$ varied $\pm 10 \%$ of optimized value. Data is as described in Fig. 1

A variety of mathematical models exist which utilize Fick's first law of diffusion to describe the transport of a solute through the skin membrane [17]. These models often relate chemical flux through skin and penetration using a permeability coefficient, combining diffusion and skin surface area components into a single constant. Previous efforts using basic physicochemical information (i.e. molecular weight) have had limited success in predicting diffusion and partitioning from the stratum corneum [14]. Our group has observed that for very lipophilic compounds $\left(\log P_{o: w}>2\right)$, the dermal partition coefficient calculation grows exponentially high (utilizing Eq. 2.3, $P_{d e r}=556$ ). Similarly, another research group 18] found difficulty in fitting $K_{p}$ for chemicals having $\log P_{o: w}>2$. Up to this point, partition coefficient equations have relied solely on molecular weight and lipophilicity. However, a different approach considering a chemical's albumin binding fraction has been proposed [14]. A description of the stratum corneum consisting of alternating layers of protein and lipids seems to provide dermal partition coefficients within expected values for highly lipophilic compounds, such as lindane. 
The relationship between parameters is often difficult to discern through strict optimization, especially when there is correlation between parameters, as is the case for $P_{d e r}$ and $D$ in Eq. 2.10 As this study used in vivo human time course data, $P_{d e r}$ and $D$ were estimated through methods that considered albumin binding (Eq. 2.8 and Eq. 2.9). This method connected both the dermal partition coefficient and the diffusion constant by taking account both the molecular size of lindane and the unbound fraction of lindane to albumin. Through varying $D$, an estimate of the steady-state permeability coefficient $K_{p}$ was obtained.

The initial estimate of $K_{p}$ using binding was approximately 10 times larger than the final estimate utilizing in vivo data for optimization $\left(1.52 \times 10^{-3}\right.$ versus $\left.1.33 \times 10^{-4}\right)$; this difference may be due to the lack of steady state permeation at the beginning of the experiment through the wiping point at six hours. The Potts-Guy equation (Eq. 2.2) yields an even higher value for $K_{p}\left(1.61 \times 10^{-2}\right)$. However, the Potts-Guy equation measures the permeability of compounds utilizing water as a vehicle for compound absorption; the experimental setup in [9] used a derivative of mineral oil as a vehicle, which has the possibility of affecting permeability of the skin. To more accurately reflect both binding of lindane to albumin as well as the effects of the vehicle on permeability, this study utilized Eq. 2.8 and Eq. 2.9 to determine an estimate of $K_{p}$.

A major goal of this work was to utilize methods of estimating dermal permeability that incorporated molecular weight and protein binding in order to more accurately represent in vivo data. This study found initial estimates of dermal coefficients, including diffusion constants and dermal partition coefficients, were only a single order of magnitude larger than the constants optimized to fit time course in vivo data, as opposed to the several orders of magnitude higher utilizing the current Potts-Guy equation. This indicates consideration of binding is essential for prediction of a steady-state permeability coefficient. This permeability coefficient can be used to determine the absorption rate for chemicals listed in the ToxCast database, thereby providing information about the overall exposure effects of a variety of different compounds. Future research is necessary to determine when mathematical estimations based solely on physio-chemical properties of the compound can predict dermal exposure without available experimental data. 


\section{Disclaimer}

This paper has been subjected to review by the National Health and Environmental Effects Research Laboratory and approved for publication. Approval does not signify that the contents reflect the views of the Agency, nor does mention of trade names or commercial products constitute endorsement or recommendation for use.

\section{Acknowledgements}

The authors would like to thank North Carolina State University and the US EPA for making this work possible. Dr. Michael F Hughes from the US EPA provided insightful comments that helped research progress. Many thanks to Dr. Paul A Lehman for unique insights into dermal experimentation. We also want to thank the following reviewers for providing insightful comments: Drs. Jane C Caldwell, Hisham A El-Masri, and Rogelio Tornero-Velez, as well as the anonymous reviewers for their helpful comments. The Research Experience for Undergraduates (REU) work was possible due to NSF grant number DMS-1063010 and NSA grant number H9823-10-1-0252. MES was partially supported through a Southern New Hampshire University 2015 Summer Research Grant.

\section{References}

\section{References}

[1] J. F. Wambaugh, R. W. Setzer, D. M. Reif, S. Gangwal, J. Mitchell-Blackwood, J. A. Arnot, O. Joliet, A. Frame, J. Rabinowitz, T. B. Knudsen, R. S. Judson, P. Egeghy, D. ValleVallero, E. A. Cohen Hubal, High-throughput models for exposure-based chemical prioritization in the expocast project, Environ. Sci. Technol. 47 (15) (2013) 8479-8488. doi:10.1021/es400482g

[2] K. K. Isaacs, W. G. Glen, P. Egeghy, M.-R. Goldsmith, L. Smith, D. Vallero, R. Brooks, C. M. Grulke, H. Özkaynak, SHEDS-HT: An integrated probabilistic exposure model for prioritizing exposures to chemicals with near-field and dietary sources, Environ. Sci. Technol. 48 (21) (2014) 12750-12759. doi:10.1021/es502513w.

[3] U.S. EPA, Lindane; Cancellation order, Fed. Regist. 71 (239) (2006) 74905-74907.

[4] C. Suñol, J. Tusell, E. Gelpí, E. Rodríguez-Farré, GABAergic modulation of lindane ( $\gamma$-hexachlorocyclohexane)-induced seizures, Toxicol. Appl. Pharmacol. 100 (1) (1989) 1-8. doi:10.1016/0041-008X(89)90086-0.

[5] J. Tusell, C. Sunol, E. Gelpi, E. Rodriguez-Farre, Relationship between lindane concentration in blood and brain and convulsant response in rats after oral or intraperitoneal administration, Arch. Toxicol. 60 (6) (1987) 432-437. doi:10.1007/BF00302386 
[6] M. Gilbert, Repeated exposure to lindane leads to behavioral sensitization and facilitates electrical kindling, Neurotoxicol. Teratol. 17 (2) (1995) 131-141. doi:10.1016/0892-0362(94)00064-K

[7] D. Loomis, K. Guyton, Y. Grosse, F. El Ghissasi, V. Bouvard, L. Benbrahim-Tallaa, N. Guha, H. Mattock, K. Straif, Carcinogenicity of lindane, DDT, and 2, 4-dichlorophenoxyacetic acid, The Lancet Oncology 16 (8) (2015) 891-892. doi:10.1016/S1470-2045(15)00081-9

[8] US Food and Drug Administration, FDA public health advisory: Safety of topical lindane products for the treatment of scabies and lice (April 2009). URL http://www.fda.gov/Drugs/DrugSafety/PostmarketDrugSaf etyInformationf orPatientsandProviders/ucm110845.htm

[9] I. Dick, P. Blain, F. Williams, The percutaneous absorption and skin distribution of lindane in man I. in vivo studies, Hum. Exp. Toxicol. 16 (11) (1997) 645-651. doi:10.1177/096032719701601103.

[10] R. Bookout Jr, C. McDaniel, D. Quinn, J. McDougal, Multilayered dermal subcompartments for modeling chemical absorption, SAR QSAR Environ. Res. 5 (3) (1996) 133-150. doi:10.1080/10629369608032985

[11] J. Stahl, F. Niedorf, M. Wohlert, M. Kietzmann, The in vitro use of the hair follicle closure technique to study the follicular and percutaneous permeation of topically applied drugs, ATLA. Alternatives to laboratory animals 40 (1) (2012) 51-57.

[12] R. O. Potts, R. H. Guy, Predicting skin permeability, Pharm. Res. 9 (5) (1992) 663-669.

[13] R. L. Cleek, A. L. Bunge, A new method for estimating dermal absorption from chemical exposure. 1. general approach, Pharm. Res. 10 (4) (1993) 497-506.

[14] L. Chen, L. Han, O. Saib, G. Lian, In silico prediction of percutaneous absorption and disposition kinetics of chemicals, Pharm. Res. 32 (5) (2015) 1779-1793. doi:10.1007/s11095-014-1575-0

[15] P. A. Lehman, A simplified approach for estimating skin permeation parameters from in vitro finite dose absorption studies, J. Pharm. Sci. 103 (12) (2014) 4048-4057. doi:10.1002/jps.24189

[16] E. L. Croom, T. J. Shafer, M. V. Evans, W. R. Mundy, C. R. Eklund, A. F. Johnstone, C. M. Mack, R. A. Pegram, Improving in vitro to in vivo extrapolation by incorporating toxicokinetic measurements: A case study of lindane-induced neurotoxicity, Toxicol. Appl. Pharmacol. 283 (1) (2015) 9-19. doi:10.1016/j.taap.2014.11.006

[17] S. Mitragotri, Y. Anissimov, A. Bunge, H. Frasch, R. Guy, J. Hadgraft, G. Kasting, M. Lane, M. Roberts, Mathematical models of skin permeability: An overview, Int. J. Pharm. 418 (1) (2011) 115-129. doi:10.1016/j.ijpharm.2011.02.023

[18] J. Bo Nielsen, J. Ahm Sø rensen, F. Nielsen, The usual suspects influence of physicochemical properties on lag time, skin deposition, and percutaneous penetration of nine model compounds, J. Toxicol. Environ. Health, Part A 72 (5) (2009) $315-323$.

[19] A. Houben, D. Slaaf, F. Huvers, P. De Leeuw, A. N. Kruseman, N. Schaper, Diurnal variations in total forearm and skin microcirculatory blood flow in man, Scand. J. Clin. Lab. Invest. 54 (2) (1994) 161-168.

[20] I. Dick, P. Blain, F. Williams, The percutaneous absorption and skin distribution of lindane in man II. in vitro studies, Hum. Exp. Toxicol. 16 (11) (1997) 652-657.

[21] K. Yamazaki, M. Kanaoka, Computational prediction of the plasma protein-binding percent of diverse pharmaceutical compounds, J. Pharm. Sci. 93 (6) (2004) 1480-1494. 